\title{
Population structure of Wolbachia and cytoplasmic introgression in a complex of mosquito species
}

\author{
Emilie Dumas ${ }^{1}$, Célestine M Atyame ${ }^{1,2}$, Pascal Milesi $^{1}$, Dina M Fonseca ${ }^{3}$, Elena V Shaikevich ${ }^{4}$, Sandra Unal ${ }^{1}$, \\ Patrick Makoundou', Mylène Weill ${ }^{1}$ and Olivier Duron ${ }^{1 *}$
}

\begin{abstract}
Background: The maternally inherited bacterium Wolbachia often acts as a subtle parasite that manipulates insect reproduction, resulting potentially in reproductive isolation between host populations. Whilst distinct Wolbachia strains are documented in a group of evolutionarily closely related mosquitoes known as the Culex pipiens complex, their impact on mosquito population genetics remains unclear. To this aim, we developed a PCR-RFLP test that discriminates the five known Wolbachia groups found in this host complex. We further examined the Wolbachia genetic diversity, the variability in the coinherited host mitochondria and their partitioning among members of the Cx. pipiens complex, in order to assess the impact of Wolbachia on host population structure.

Results: There was a strong association between Wolbachia and mitochondrial haplotypes indicating a stable co-transmission in mosquito populations. Despite evidence that members of the $C$. pipiens complex are genetically distinct on the basis of nuclear DNA, the association of Wolbachia and mtDNA with members of the $C x$. pipiens complex were limited. The Wolbachia wPip-I group, by far the most common, was associated with divergent Cx. pipiens members, including Cx. quinquefasciatus, Cx. pipiens pipiens form pipiens and Cx. pipiens pipiens form molestus. Four other wPip groups were also found in mosquito populations and all were shared between diverse Cx. pipiens members.

Conclusion: This data overall supports the hypothesis that wPip infections, and their allied mitochondria, are associated with regular transfers between $C x$. pipiens members rather than specific host associations. Overall, this is suggestive of a recent and likely ongoing cytoplasmic introgression through hybridization events across the Cx. pipiens complex.
\end{abstract}

Keywords: Endosymbiosis, Wolbachia, Cytoplasmic introgression, Culex pipiens complex

\section{Background}

Symbiotic associations with the intracellular bacterium Wolbachia are extremely widespread in insects [1-3]. Wolbachia is typically maternally inherited through the egg cytoplasm and has evolved a variety of interactions with its hosts, exerting subtle effects such as manipulation of host reproduction or protection against natural enemies [4-6]. Each of these effects is advantageous to infected females and thus enables Wolbachia to spread rapidly

\footnotetext{
* Correspondence: olivier.duron@univ-montp2.fr

'Institut des Sciences de l'Evolution, UMR5554 CNRS, Université Montpellier 2, 34095 Montpellier cedex 05, France

Full list of author information is available at the end of the article
}

through insect populations. These effects are also of ecological and evolutionary importance to the particular host species that is infected, potentially inducing reproductive isolation or driving changes in sexuality [4-6].

Mosquitoes of the Culex pipiens complex have long been recognized to exhibit a great variability of effects associated with Wolbachia. In this host, Wolbachia, known as $w$ Pip, is associated with cytoplasmic incompatibility (CI), a sperm-egg incompatibility between infected males and uninfected females, so that infected females have a reproductive advantage [7-9]. Apart from this simple case, $\mathrm{CI}$ has been also observed in a number of cases between males and females carrying incompatible $w$ Pip strains

\section{Biomed Central}


[9-12]. Five housekeeping genes developed for Wolbachia multilocus strain typing (MLST) [13] and the Wolbachia surface protein gene $w s p$ present no variation among these $w$ Pip strains, showing that they delineate a recent and monophyletic clade into the B Wolbachia supergroup [14]. The recent examination of fast evolving markers, such as the ank 2 and $p k 1$ genes encoding proteins with ankyrin (ANK) motifs, revealed the existence of more than 100 genetically distinct $w$ Pip strains $[9,11,14]$, belonging to five distinct subclades and further referred as $w$ Pip-I to $w$ Pip-V groups [14]. Since their molecular characterization, several recent studies have further showed that some $w$ Pip strains are mutually incompatible but also that some others, although genetically distinct, are fully compatible $[9,11,14]$. Meanwhile, $w$ Pip has also emerged as a conditional mutualist that protects $C x$. pipiens against mortality induced by the avian malaria parasite Plasmodium relictum [15]. Overall, these studies revealed that $w$ Pip is an important associate of most mosquitoes in the Cx. pipiens complex potentially driving its evolution.

How $w$ Pip infections interact with the genetic structure within the $C x$. pipiens complex remains still unclear. Mosquitoes in this complex have a global distribution in all temperate and tropical regions, with a recent history of association with human migration [16,17]. The complex encompasses a group of genetically closely related taxa with distinct behavioural and physiological traits that greatly influence their distribution. The most obvious variable traits include larval habitat preference, vertebrate feeding pattern, mating behaviour, gonotrophic development and ability to enter into diapause during the winter [17]. Despite these differences, members of the $C x$. pipiens complex have controversial taxonomic statuses (including subspecies, forms or biotypes delineations) and, even though they are genetically distinct, they remain difficult to separate morphologically. Four species, or subspecies according to the authors, are frequently recognized as members of the complex: $C x$. pipiens, $C x$. quinquefasciatus, $C x$. australicus, and $C x$. globocoxitus, as well as two subspecies: Cx. pipiens pipiens in Europe and North and South Africa, and $C x . p$. pallens, in Asia. In addition, two sympatric forms, pipiens and molestus, are also encountered in $C x . p$. pipiens in the Northern hemisphere. $C x$. quinquefasciatus, commonly known as the southern house mosquito, exists all across the tropics and the lower latitudes of temperate regions. $C x$. australicus and $C x$. globocoxitus are restricted to Australia and are poorly known. Although the exact taxonomic status of the $C x$. pipiens members remains controversial, their close evolutionary association has been repeatedly supported by genetic analyses and the relative abundance of hybrids in areas where distributions overlap [18-22]. Remarkably, whilst $w$ Pip was never detected in $C x$. australicus and $C x$. globocoxitus, infection frequency was near or at fixation in almost all populations of both $C x$. pipiens and $C x$. quinquefasciatus [8,23-25]. When first described, any of the five $w$ Pip groups were found associated within given infected members of the $C x$. pipiens complex [14]. However, only a limited number of $C x$. pipiens and $C x$. quinquefasciatus laboratory lines have been examined, making this result difficult to interpret. This issue is of special importance since Wolbachia may either produce reproductive isolation between host populations infected with different Wolbachia strains, or reinforce an existing divergence by selecting for pre-mating isolation mechanisms $[4,5]$. The presence of closely related host taxa within the Cx. pipiens complex is thus a relevant system to test such a hypothesis.

Here we have approached this issue by undertaking an extensive screening for the presence, the diversity and the partitioning of $w$ Pip infections in natural populations of the four main members of the $C x$. pipiens complex (Cx. quinquefasciatus, $C x$. $p$. pipiens form pipiens, $C x . p$. pipiens form molestus and $C x . p$. pallens), spanning 118 natural populations and 64 laboratory lines. With this aim, we characterized each $w$ Pip individual infection using two to five Wolbachia markers and identified $C x$. pipiens members using diagnostic nuclear markers, including microsatellites for a subsample of populations. Since Wolbachia and host mitochondria are co-transmitted in egg cytoplasm and therefore are in linkage disequilibrium, we also examined the mitochondrial (mtDNA) diversity through the sequencing of one to three mtDNA markers. Using this approach, we thus attempted to infer the contribution of Wolbachia in shaping the genetic diversity within the $C x$. pipiens complex.

\section{Methods}

\section{Mosquito collection}

We examined mosquitoes from Europe, Asia, Oceania, Africa and America belonging to the four members of the $C x$. pipiens complex: $C x$. p. pipiens form pipiens, $C x . p$. pipiens form molestus, $C x . p$. pallens as well as $C x$. quinquefasciatus, hereinafter respectively referred to as pipiens, molestus, pallens and quinquefasciatus (Additional file 1: Table S1). The collection encompasses both natural populations (mostly sampled during the period 1990 to 2012) and isofemale laboratory lines (derived from field specimens collected from 1950 to 2011). Each laboratory line descended from a single female founder and was further considered as a single individual. All specimens were stored in liquid nitrogen or in $70-95 \%$ ethanol, at room temperature or in a freezer at $-20^{\circ} \mathrm{C}$ until examined.

\section{Molecular typing}

The $w$ Pip infections were genotyped and assigned to one group (wPip-I to $w$ Pip-V) using a series of specific PCR- 
RFLP (restriction fragment length polymorphism) assays based on two ANK Wolbachia markers, ank2 and pk1 (Additional file 1: Table S2 and Figure S1). Hinfl digestion of the ank2 PCR products allowed discrimination of five alleles ( $\mathrm{a}$ to e): a (one RFLP fragment: $313 \mathrm{bp}$ ), b (217, 195, 98 bp), c (293, 217 bp), d (217, 195 bp) and e (415 bp). TaqI digestion of the $p k 1$ PCR products allowed discrimination of four specific $w$ Pip alleles (alleles a and e have the same fragment size): a/e (903, $430 \mathrm{bp}), \mathrm{b}(669,665 \mathrm{bp}), \mathrm{c}(851,498 \mathrm{bp})$ and d (497, 251, $107 \mathrm{bp})$. The $p k 1$ a and e alleles were next distinguished using a digestion of the $p k 1$ PCR products with PstI: a (903, 303, 141 bp) and e (903, 430 bp). For a subsample of specimens, three additional Wolbachia markers were sequenced: the DNA mismatch repair protein gene $M u t L$, the putative secreted protein gene GP15 (also called $\mathrm{VrlC}$ ) and the regulatory protein gene RepA (Additional file 1: Table S2). Four (ank2, MutL, GP15 and $\operatorname{RepA}$ ) of these five Wolbachia genes were present in one single copy in the $w$ Pip (Pel) genome while the fifth ( $p k 1)$ is present in three identical copies (Additional file 1: Table S2; see [14] and [26], for details). A total of 5 Wolbachia genes, encompassing 7 distinct loci with a wide distribution along the $w$ Pip (Pel) chromosome, were thus examined. None of these genes was amplified from Wolbachia-free Cx. pipiens lines, which confirmed their Wolbachia origin.

DNA was extracted from individual mosquitoes using a CetylTrimethylAmmonium Bromide (CTAB) [27] or phenol/chloroform protocols [28]. All PCR amplification conditions were: $5 \mathrm{~min}$ at $94^{\circ} \mathrm{C}$, followed by $30-40$ cycles of $94^{\circ} \mathrm{C}$ for $30 \mathrm{~s}, 50^{\circ} \mathrm{C}-58^{\circ} \mathrm{C}$ for $30 \mathrm{~s}$, and $72^{\circ} \mathrm{C}$ for 1 to 1.5 min depending on the fragment size (detailed on Additional file 1: Table S2). Digestion of PCR products were performed following manufacturer's instructions. The QIAquick gel extraction kit (QIAGEN, Valencia, CA) was used to purify the PCR products for sequencing. Sequences were obtained directly from purified products on an ABI Prism 3130 sequencer using the BigDye Terminator Kit (Applied Biosystems).

The $C x$. pipiens mtDNA haplotypes were determined through the sequencing of an $852 \mathrm{bp}$ fragment from the cytochrome $b$ (cytb) gene (Additional file 1: Table S2). For a subsample of specimens, we also obtained partial sequences of two additional mtDNA markers: the NADH dehydrogenase subunit 2 (ND2, $1160 \mathrm{bp})$ and 5 (ND5, 1132 bp) (Additional file 1: Table S2).

The four Cx. pipiens taxa were identified using nuclear DNA of specimens. We included in this study some populations available from previous studies for which $C x$. pipiens taxa were determined using 7 to 12 microsatellite loci and/or sequence variation in an intron of the acetylcholinesterase-2 (ace-2) gene (Additional file 1: Table S1). The taxa of new $C x$. pipiens populations were identified using a combination of the PCR-RFLP on ace-2 $[29,30]$ and a multiplex-PCR assay based on the flanking region of a microsatellite locus (CQ11; see [31] for more details) (Additional file 1: Table S1 and Table S2). Specimens from these new populations were considered as hybrids when they showed a heterozygous genotype at ace-2 or CQ11 loci.

\section{Data analyses}

Sequence alignments were carried out using ClustalW [32] and corrected using MEGA [33]. The GBLOCKS program [34] with default parameters was used to remove poorly aligned positions and to obtain unambiguous sequence alignments. The evolutionary model most closely fitting the sequence data was determined using Akaike information criterion with the MEGA program [33]. Phylogenetic analyses were conducted using maximum likelihood (ML) and maximum-parsimony (MP) in MEGA [33]. ML phylogenies were constructed based upon unambiguously aligned sites using the Tajima-Nei model of nucleotide substitution, assuming that nucleotide frequencies deviate substantially from 0.25 [35]. MP phylogenies were constructed using the close-neighbourinterchange method [36]. Bootstrap probabilities were calculated by generating 500 bootstrap replicates. New sequence data were deposited in GenBank (accession numbers KC686688- KC686692).

The microsatellite data was first examined for compliance with Hardy-Weinberg equilibrium, and then pairwise $F_{\mathrm{ST}}$ values and their significance were obtained using GENEPOP v4.2 [37]. We assigned specimens to genetic clusters with a maximum likelihood algorithm implemented in the program Structure 2.3.4 [38]. This method combines all the individual multilocus genotypes and separates them into $\mathrm{K}$ distinct clusters. We used the admixture model taking into account correlated allele frequencies between populations with 10,000 burn-in steps and 100,000 runs as MCMC (Markov chain Monte Carlo) parameters to assign specimens to genetic clusters. The degree of admixture, alpha, was inferred from the data (if alpha approaches zero most individuals are not admixed). With this parameter set, the geographic origin of each specimen is not considered but the number of clusters (from $K=1$ to $K=7$ ) is decided a priori for each run. To assess the consistency of the analysis we performed an exhaustive comparison of 10 runs for each $\mathrm{K}$ to assess the robustness of the results. We also performed the method described in Evanno et al. [39] to confirm that the true number of clusters explaining our data was detected.

The significance of non-random associations between $w$ Pip/mtDNA, $w$ Pip/Culex pipiens taxa or mtDNA/Culex pipiens taxa was estimated using an exact test procedure (Fisher exact test) implemented in GENEPOP [37]. 


\section{Results}

Polymorphism of wPip infections

We assayed for the presence and the diversity of Wolbachia in 1935 specimens from 118 natural populations and 64 isofemale laboratory lines (Additional file 1: Table S1). Specific ank2 and $p k 1$ PCR assays indicated the occurrence of infection by Wolbachia in all the examined specimens, showing that infection is fixed in all $C x$. quinquefasciatus, $C x$. p. pipiens form pipiens, $C x$. $p$. pipiens form molestus and $C x$. p. pallens populations examined here.

We further used the ank2 and $p k 1$ allelic profiles to assign a $w$ Pip group to each specimen (Table 1). We found in our collection the five known ank 2 alleles and the five known $p k 1$ alleles. Tests for intergenic recombination revealed significant linkage disequilibrium (LD) for ank2 and $p k 1$ (Fisher exact test, $P<10^{-5}$ ): alleles at these loci are not randomly associated showing that they are stably co-transmitted within the $w$ Pip chromosome. Hence, the ank2 and $p k 1$ allelic profiles are congruent and allowed unambiguously attributing the $w$ Pip group to $1836(94.9 \%)$ of 1935 specimens (Table 1). For the 99 (5.1\%) remaining specimens, ank2 and pk1 allelic profiles were not congruent with known haplotypes (Table 1), suggesting that these two loci had undergone recombination. To assign these 99 specimens to a $w$ Pip group, three additional Wolbachia markers were then sequenced: MutL, GP15 and RepA. Three to thirteen alleles were detected for each marker and led to the identification of 30 new $w$ Pip haplotypes from the 99 unassigned specimens (Table 1). Some of these alleles are null alleles: some specimens did not amplify at one of the loci (because of either mutation in the primer sites or gene deletion) showing that their $w$ Pip infections were genetically distinct from other known haplotypes. Positive amplifications of other loci ( $p k 1$, ank2) from samples with null alleles at either MutL, GP15 or RepA loci indicate satisfactory DNA template quality in these cases. Phylogenetic tree using ank2, pk1, MutL, GP15 and RepA concatenated sequences (5624 bp) showed that almost all new $w$ Pip haplotypes fall into one of the five $w$ Pip groups (Figure 1). Overall, the use of the three additional Wolbachia markers allowed the assignment to a $w$ Pip group to 96 of the 99 remaining specimens, with only two haplotypes (\#29 found in two specimens and \#30 in one specimen) remaining not assigned.

Taking into account PCR-RFLP and phylogenetic grouping, we thus found that 823 specimens (42.5\%) were infected by the $w$ Pip-I group, $326(16.9 \%)$ by $w$ PipII, 414 (21.4\%) by $w$ Pip-III, 261 (13.5\%) by $w$ Pip-IV, 108 $(5.6 \%)$ by $w$ Pip-V and $3(0.1 \%)$ by undetermined $w$ Pip groups (Table 1). We found no evidence of specimens infected by more than one $w$ Pip group: only one allele per gene was observed for each DNA sample. The subsequent sequencing of ank 2 and $p k 1$ PCR products obtained from a subsample of specimens confirmed the observed RFLP profiles, with no double peaks (indicative of multi-infection) in electropherograms.

\section{Geographic distribution of wPip diversity}

The diversity of $w$ Pip showed an important spatial variation over the distribution area of the $C x$. pipiens complex (Figures 2A, 2B, Additional file 1: Table S1). Two $w$ Pip groups were found to dominate wide geographic regions: only $w$ Pip-I was found in Sub-Saharan Africa, South America and Southeast Asia, and only wPip-III was observed in North America. The three other groups, $w$ Pip-II, $w$ Pip-IV and $w$ Pip-V, were less common. While $w$ Pip-II and $w$ Pip-V were confined to Western Europe and Asia, respectively, with very few exceptions, $w$ Pip-IV was more disseminated and was sporadically found in Europe, North Africa and Asia. Generally, only one $w$ Pip group was found per geographic region indicating a regional homogeneity of Wolbachia infections (Figure 2A), but a contrasting picture emerged in Europe where all five $w$ Pip groups were found (Figure $2 \mathrm{~B}$ ). Given that Europe is oversampled (709 individuals from 66 populations; Additional file 1: Table S1) relative to other parts of the world, the $w$ Pip diversity observed there could be simply a function of a higher degree of sampling effort. However, some regions have been also extensively sampled and still revealed fewer $w$ Pip groups (i.e. Africa: 569 individuals from 51 populations but only two $w$ Pip groups). Thus, oversampling does not seem to be a likely explanation for the great $w$ Pip diversity found in Europe.

In most cases, only one $w$ Pip group was detected per population: $88(74.6 \%)$ of the 118 natural populations harboured only one $w$ Pip group while, in the remaining $30(25.4 \%)$, two to three $w$ Pip groups per population were observed. Twenty-two of the 30 populations were located in Europe and half (18 of 30) harboured $C x$. pipiens individuals infected either by $w$ Pip-II or $w$ Pip-III. Aside from the $w$ Pip-II/wPip-III mixed populations found in Western Europe, at least five other geographic contact zones between $w$ Pip groups exist: in the North (wPip-II/wPip-IV) and in the South of Italy ( $w$ Pip-I/ $w$ Pip-IV), in North Africa (wPip-I/wPip-IV), in South America ( $w$ Pip-I/wPip-III) and Eastern Asia ( $w$ Pip-I/ $w$ Pip-V) (Figures 2A, 2B).

\section{Association of wPip groups with mtDNA and members of the $C x$. Pipiens complex}

To investigate the association between $w$ Pip groups and mtDNA variation, we sequenced the $c y t b$ gene (that have been shown polymorphic in the $C x$. pipiens complex, see [14]) in a subsample of 184 specimens from $101 C x$. pipiens natural populations and 44 isofemale laboratory lines. The $c y t b$ gene displayed low variability and eleven 
Table 1 Allelic profiles of Wolbachia genes in the five wPip groups

wPip group Haplotypes o w Haplotypes

\begin{tabular}{|c|c|c|c|c|c|c|c|}
\hline WPIp group & WPIp Hapiotypes & 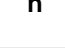 & ank2 & pk1 & GP15 & MutL & $\operatorname{RepA}$ \\
\hline \multicolumn{8}{|l|}{ WPip-I } \\
\hline & wPip-I known haplotypes & 821 & a & a & a & a & $a, b$ \\
\hline & $\# 1$ & 1 & $d$ & a & a & a & a \\
\hline & \#2 & 1 & $b$ & a & ND & ND & ND \\
\hline
\end{tabular}

Population numbers

WPip-ll known haplotypes $276 \quad$ e $\quad c \quad b, e, f \quad b, c, d \quad a$

64/ 160-107/ 115-117/ 121-122/ 130-132/ 137-145/ 147/ 152-155/ 157/ 159-160/ 165-167/ 179-182

\begin{tabular}{|c|c|c|c|c|c|}
\hline \#3 & 1 & a & c & $j^{*}$ & c \\
\hline$\# 4$ & 1 & $\mathrm{a}$ & c & $f$ & - \\
\hline$\# 5$ & 2 & a & c & $\mathrm{b}$ & c \\
\hline$\# 6$ & 3 & a & c & $\mathrm{b}$ & b \\
\hline$\# 7$ & 3 & b & c & c & ND \\
\hline \#8 & 1 & $b$ & c & c & e \\
\hline \#9 & 3 & b & $c$ & ND & c \\
\hline \#10 & 9 & $b$ & $c$ & ND & ND \\
\hline \#11 & 4 & b & c & $h^{*}$ & c \\
\hline \#12 & 3 & $b$ & c & $i^{*}$ & b \\
\hline \#13 & 1 & $b$ & c & $k^{*}$ & c \\
\hline \#14 & 7 & $b$ & c & $h^{*}$ & c \\
\hline \#15 & 1 & $b$ & c & $c$ & - \\
\hline \#16 & 10 & $b$ & C & k & c \\
\hline \#17 & 1 & c & c & $d$ & - \\
\hline
\end{tabular}

wPip-III

wPip-III known haplotypes 401

$\begin{array}{lllllll}\# 18 & 1 & \text { a } & \text { b } & - & - & - \\ \# 19 & 1 & c & \text { b } & - & - & \text { b } \\ \# 20 & 6 & \text { d } & \text { b } & \text { c } & \text { b } & \text { b } \\ \# 21 & 1 & \text { e } & \text { b } & \text { c } & - & \text { b } \\ \# 22 & 1 & \text { e } & \text { b } & \text { i } & \text { ND } & \text { a } \\ \# 23 & 1 & \text { e } & \text { b } & \text { ND } & \text { ND } & \text { ND } \\ \# 24 & 1 & \text { e } & \text { b } & \text { f } & \text { c } & \text { a } \\ \# 25 & 1 & \text { e } & \text { b } & \text { c } & \text { e } & \text { b }\end{array}$

18/ 72-76/ 78-89/ 92-94/ 96-99/131/ 134/ 136/ 140/ 144-145/ 148-152/ 154-156/ 158-161/ 165-167/ 179/ 182

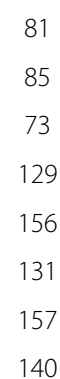


Table 1 Allelic profiles of Wolbachia genes in the five wPip groups (Continued)

WPip-IV

wPip-IV known haplotype

\#26

wPip-V

$260 \quad c \quad d \quad d$

c a

a

51/ 90/ 100-104/118/121/ 125-129/131/133/135/ 169-174

129

$\begin{array}{ccccccc}\text { WPip-V known haplotype } & 78 & \text { d } & \text { e } & \text { g } & \text { f } & \text { a } \\ \# 27 & 19 & \text { a } & \text { e } & \text { g } & \text { c } & \text { a } \\ \# 28 & 11 & \text { b } & \text { e } & \text { g } & \text { a } & \text { a }\end{array}$

\#29

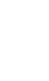

(1)

undetermined

$$
\text { \#29 }
$$

$$
2 \text { b } \quad \text { a } \quad c \quad c \quad c \quad b
$$

New wPip haplotypes are identified by \#number. Allelic profiles for GP15, MutL and RepA loci were determined for all new wPip haplotypes and for a subsample of specimens for the known wPip haplotypes. ND, allele not determined (DNA shortage); Dash, null allele (no PCR product); Asterisk, new allele. 


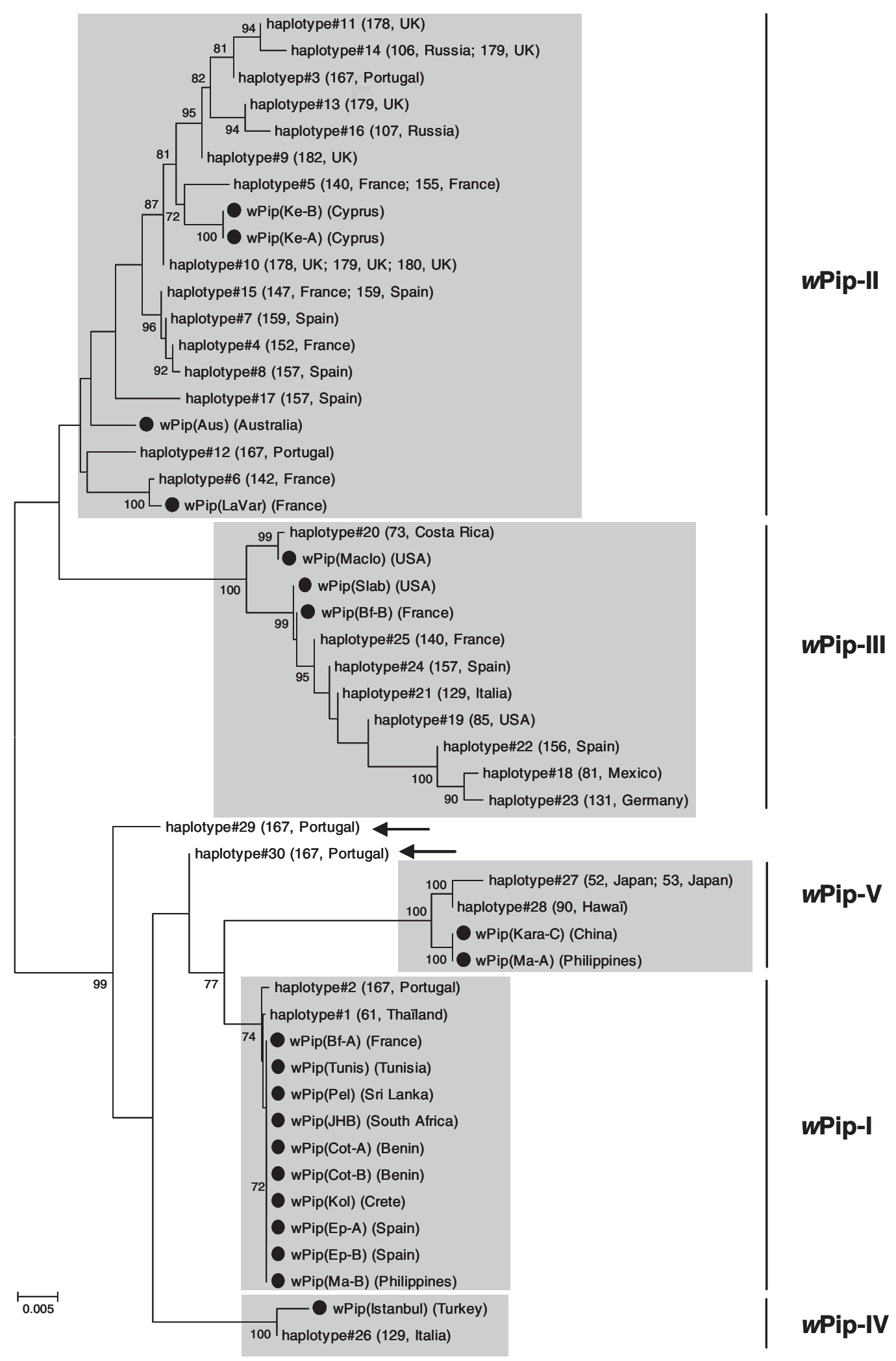

Figure 1 (See legend on next page.) 
(See figure on previous page.)

Figure 1 wPip haplotypes phylogeny constructed using Maximum Parsimony method based on concatenated sequences of ank2, pk1, MutL, GP15 and RepA genes. Known WPip strains haplotypes are marked by full circles. Arrows show the two non-assigned haplotypes.

Numbers on branches indicate percentage bootstrap support (500 replicates). Only bootstrap values $>70$ were shown. The scale bar indicates the number of substitutions. Numbers in brackets correspond to locality numbers in Additional file 1: Table S1.

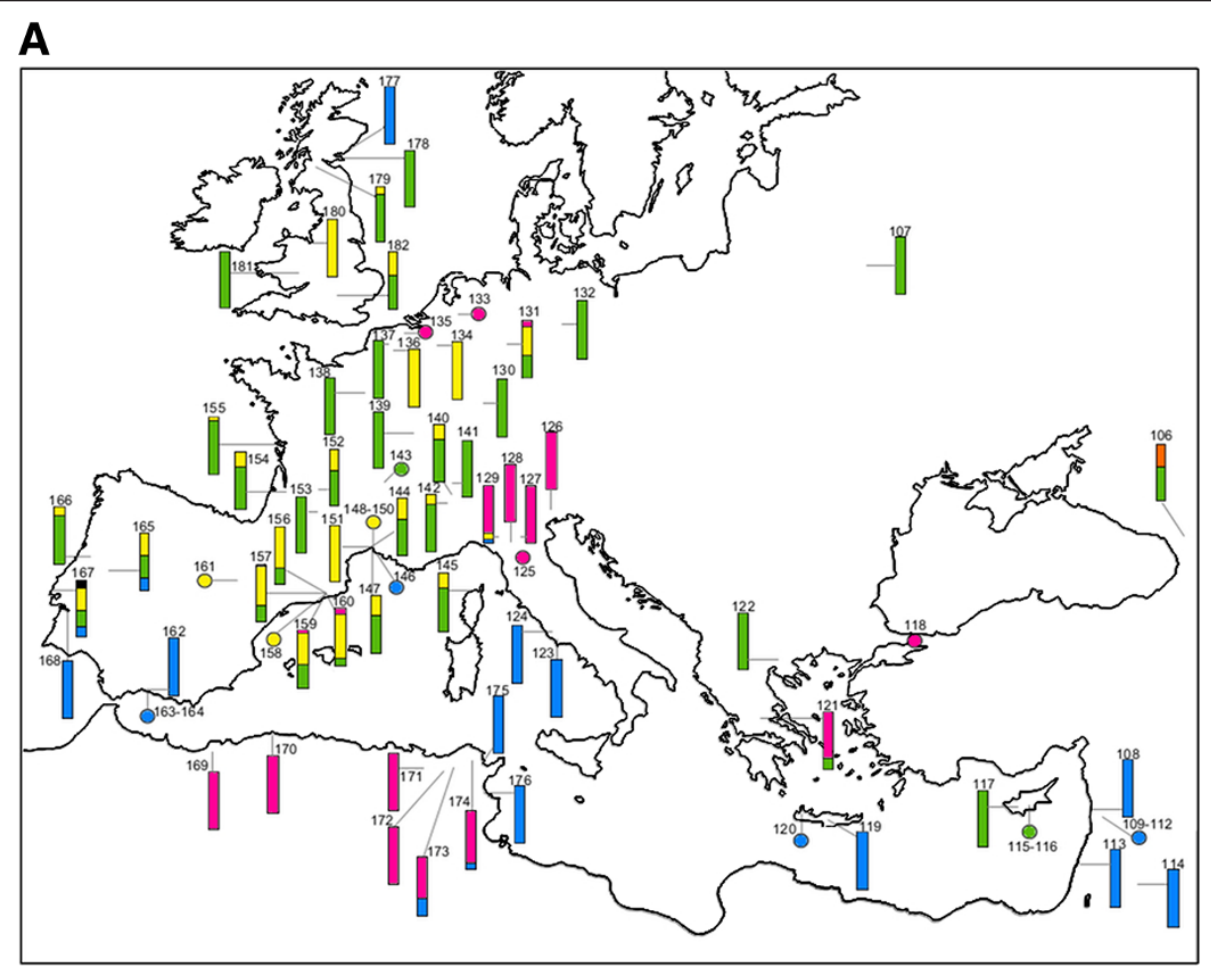

\section{B}

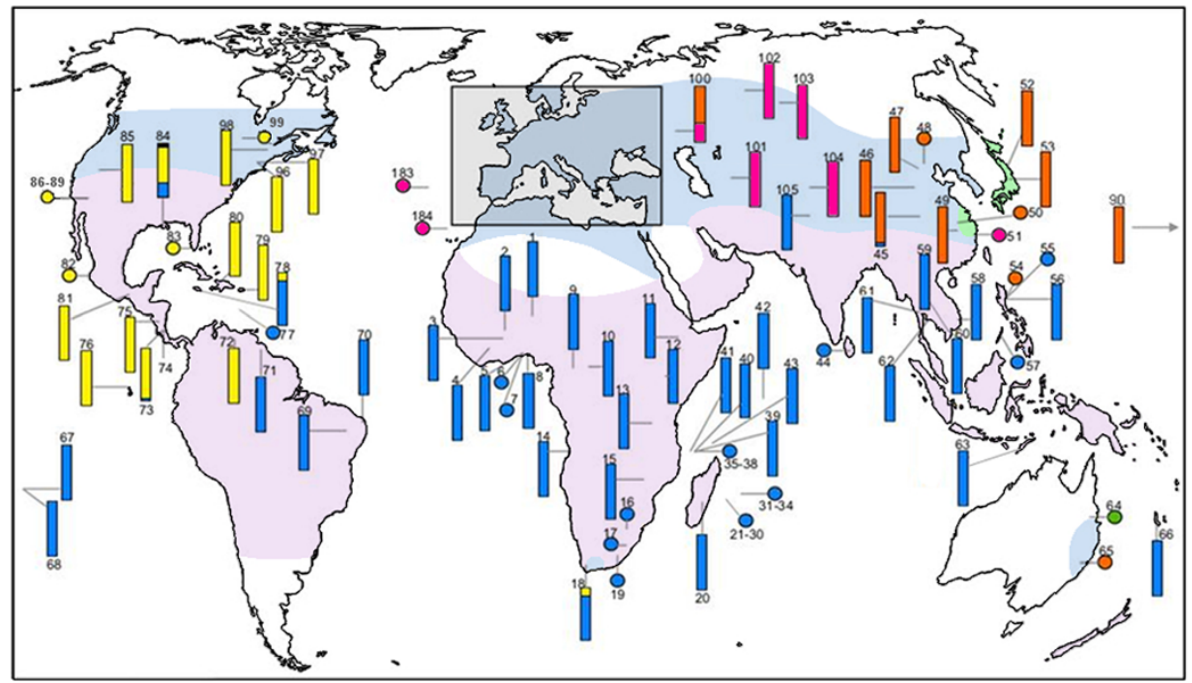

Figure 2 Geographic distribution of wPip groups in the World (A) and in Europe (B). Numbers in the maps correspond to locality numbers in Additional file 1: Table S1. Bars and dots represent natural populations and laboratory strains, respectively. The bars show the prevalence of wPip group: blue, wPip-I infection; green, wPip-II; yellow, wPip-III; pink, wPip-IV; orange, wPip-V; black, undetermined group. On Figure 2A, Culex pipiens pipiens form pipiens and Culex pipiens pipiens form molestus = light blue; $C$. $p$. pallens = light green and $C X$. quinquefasciatus $=$ light pink (modified after Farajollahi et al. 2011). On Figure 2B, both Cx. p. pipiens form pipiens and Cx. p. pipiens form molestus are widespread in Europe. Details on sample size, prevalence and $C$. pipiens complex taxa are given in Additional file 1: Table $\mathbf{S 1 .}$ 
haplotypes with only ten variable nucleotide positions throughout the 852 bp cytb fragment (ca. 99.5\% of pairwise identity) were obtained (Additional file 1: Table S3). Pairwise tests revealed significant LD for $w$ Pip groups and cytb haplotypes $\left(P=10^{-5}\right)$ with a clear pattern of $c y t b$ haplotype specificity to $w$ Pip groups (Table 2 ). According to this association and following previous study [14], cytb haplotypes were partitioned in five mtDNA groups (mtDNA-1 to 5 associated to $w$ Pip-I to $\mathrm{V}$ groups, respectively; Table 2).

When analysing the congruence between the five mtDNA and $w$ Pip groups, very little incongruence were observed. For instance, although the mtDNA-1 group contains $65 w$ Pip-I-infected specimens, seven specimens infected with $w$ Pip-V were also found in this group (Table 2). A very similar pattern was found for five other specimens: one $w$ Pip-I-infected specimen was found in the mtDNA-4 group (otherwise associated to $20 w$ PipIV-infected specimens), one $w$ Pip-II-infected specimen in the mtDNA-3 group (associated to $44 w$ Pip-IIIinfected specimens), and $3 w$ Pip-III-infected specimens in the mtDNA-2 group (associated to $39 w$ Pip-IIinfected specimens). To improve our understanding of this incongruence, two additional mtDNA genes (ND2 and ND5) were sequenced and phylogenetic analyses were conducted using $c y t b, N D 2$ and ND5 concatenated genes (2549 bp; Additional file 1: Figure S2). Only the seven $w$ Pip-V-infected specimens found associated with the mtDNA-1 group on the basis of $c y t b$ sequence were analysed since not enough DNA was available from the five remaining specimens. These analyses also included the two unassigned $w$ Pip haplotypes (\#29 and \#30) described above. The resulting phylogenetic tree does not group the seven incongruent specimens infected by $w$ Pip-V within the extended mtDNA-1 group, and they can not be assigned to a specific mtDNA group. These results suggest that the primary $w$ Pip-mtDNA incongruence observed in these specimens is likely due to low polymorphism in the mitochondrial gene studied here (see Additional file 1: Table S3). The two unassigned wPip haplotypes, \#29 and \#30, were clearly associated with mtDNA-3 and mtDNA-2, respectively. The strong association between mtDNA and $w$ Pip infection presented above indicates that these two haplotypes could be assigned with a low risk of error to $w$ Pip-II and $w$ PipIII groups.

To investigate the association of $w$ Pip groups with taxa within the $C x$. pipiens complex, we examined the partitioning of $w$ Pip groups among $409 C x$. pipiens specimens assigned to one of four $C x$. pipiens complex taxa: quinquefasciatus $(\mathrm{n}=201,49.1 \%)$, pipiens $(\mathrm{n}=118,28.8 \%)$, molestus $(\mathrm{n}=64,15.7 \%)$, and pallens $(\mathrm{n}=20,4.9 \%)$ as well as a few hybrids $(n=6,1.5 \%)$ (Additional file 1: Table S1). The observed geographic distribution of our specimens was very similar to the known distribution of the $C x$. pipiens taxa $[17,30]$ with quinquefasciatus widely present in tropical areas, pipiens and molestus in Europe, North Africa, Middle East and North America, and pallens confined to East Asia (Figures 2A, 2B). The association between $C x$. pipiens taxa and $w$ Pip groups was highly significant (Fisher exact test, $P<10^{-4}$ ), as shown with quinquefasciatus that appeared mainly infected with the

Table 2 Mitochondrial (cytb) haplotypes and partitioning between wPip groups

\begin{tabular}{|c|c|c|c|c|c|c|c|c|}
\hline mtDNA group & cytb haplotype & $\mathrm{n}$ & wPip-I & wPip-II & wPip-III & wPip-IV & wPip-V & Undetermined \\
\hline \multicolumn{9}{|l|}{ mtDNA-1 } \\
\hline & $\# 1$ & 1 & 1 & 0 & 0 & 0 & 0 & 0 \\
\hline & $\# 2$ & 50 & 43 & 0 & 0 & 0 & 7 & 0 \\
\hline & \#3 & 3 & 3 & 0 & 0 & 0 & 0 & 0 \\
\hline & \#4 & 18 & 18 & 0 & 0 & 0 & 0 & 0 \\
\hline \multicolumn{9}{|l|}{$m t D N A-2$} \\
\hline & \#5 & 43 & 0 & 39 & 3 & 0 & 0 & 1 \\
\hline \multicolumn{9}{|l|}{ mtDNA-3 } \\
\hline & \#6 & 43 & 0 & 1 & 41 & 0 & 0 & 1 \\
\hline & \#7 & 3 & 0 & 0 & 3 & 0 & 0 & 0 \\
\hline \multirow[t]{2}{*}{$m t D N A-4$} & \#8 & 5 & 1 & 0 & 0 & 4 & 0 & 0 \\
\hline & $\# 9$ & 16 & 0 & 0 & 0 & 16 & 0 & 0 \\
\hline \multicolumn{9}{|l|}{ mtDNA-5 } \\
\hline & $\# 10$ & 1 & 0 & 0 & 0 & 0 & 1 & 0 \\
\hline & $\# 11$ & 1 & 0 & 0 & 0 & 0 & 1 & 0 \\
\hline & Total & 184 & 66 & 40 & 47 & 20 & 9 & 2 \\
\hline
\end{tabular}


wPip-I group (133 of 201 individuals), pipiens with $w$ Pip-II (55 of 118) and pallens with $w$ Pip-V (19 of 20) (Table 3). However, the pattern is less clear for molestus that showed a more balanced prevalence of $w$ Pip groups with $w$ Pip-I, $w$ Pip-II and $w$ Pip-III more or less equally prevalent in molestus (each infecting 15 to 20 of 64 individuals). Overall, the association $w$ Pip/Culex pipiens taxa is thus far from exclusive since no $w$ Pip group was unique to a particular $C x$. pipiens member: each $C x$. pipiens taxa harbours two to five different $w$ Pip groups. For instance, pipiens harbours infections of all five $w$ Pip groups and quinquefasciatus of three $w$ Pip groups ( $w$ Pip-I, $w$ Pip-III and $w$ Pip-V).

Alternatively, the heterogeneity of $w$ Pip infections among $C x$. pipiens members could be based on potential artefacts from molecular typing: for some samples, taxa identification was done through the characterization of two nuclear markers, ace-2 and CQ11, an approach that could fail to detect hybrid individuals. To address this potential bias we refined our analysis by restricting our analyses to a subsample of 113 specimens from 12 populations (encompassing populations of quinquefasciatus: populations 12, 63, 76, 78, 84 and 90 in Table S1, pipiens: 130 and 181, molestus: 114 and 131, pallens: 52 and 53) that were typed with microsatellite loci. Although these mosquito populations were originally typed using 7-12 microsatellite loci, the loci used were however different depending on what $C x$. pipiens taxa and studies they were for. Further analyses were thus conducted using four microsatellite loci (GT4, CxpGT12, GT46 and CQ26; [40,41]) for which genotypes were available for all specimens. None of these loci had significant heterozygote deficits/excess in the examined populations, fitting with Hardy-Weinberg assumptions. A pair-wise $F_{\mathrm{ST}}$ comparison revealed significant differentiation between mosquito populations belonging to different taxa in all cases $\left(F_{\mathrm{ST}}\right.$ values ranged from 0.165 to 0.660 ; Additional file 1: Table S4), while within taxa the $F_{\mathrm{ST}}$ values ranged from only 0.012 to 0.251 and were in some cases not different from zero (Additional file 1: Table S4), indicating that gene flow is more important within than between $C x$. pipiens taxa. The result of the mosquito genetic structure analysis further separates the individuals into five distinct clusters analogous to the Culex taxa assignation (Figure 3). Indeed, the clustering separate pallens, pipiens, molestus, quinquefasciatus from America and quinquefasciatus from Africa and Asia. This clustering indicates a low hybrid rate (alpha $\approx 0.03$ ) indicating that most individuals are not admixed and come from a cluster (Figure 3). The assignment of individuals using microsatellite data was in agreement with our primary assignment, although it separates quinquefasciatus populations in two distinct clusters depending on their geographic origins. Same as above, the association between $w$ Pip groups and $C x$. pipiens members was still significant (Fisher exact test, $P<10^{-4}$ ) and also confirmed that this association is not exclusive since at least four $w$ Pip groups are shared by different $C x$. pipiens members (Figure 3). The presence of shared $w$ Pip groups between $C x$. pipiens members thus suggests that $w$ Pip infections undergo repeated transfers between quinquefasciatus, pipiens, molestus and pallens.

Finally, the association between mtDNA and $C x$. pipiens members was assayed in 166 individuals (Table 4). As observed with $w$ Pip groups, there was a significant association between mtDNA and $C x$. pipiens members (Fisher exact test, $P<10^{-4}$ ), but not exclusive: although mtDNA-1 group was common in quinquefasciatus (59 of 72 individuals) and mtDNA-2 group was common in pipiens (35 of 67), no mtDNA group was specific to a particular $C x$. pipiens member (Table 4). As could be expected due to LD between $w$ Pip and mtDNA, the mtDNA distribution thus mirrored the $w$ Pip distribution across the $C x$. pipiens complex, showing that they form together a single cytoplasmic unit. Overall, these results show that $w$ Pip and mtDNA share a joint evolutionary history, subtly different to the one of nuclear DNA and thus to the evolutionary histories of $C x$. pipiens members.

\section{Discussion}

We sampled four evolutionarily closely related mosquito taxa within the Cx. pipiens complex for Wolbachia

Table 3 Partitioning of wPip groups among $C x$. pipiens taxa

\begin{tabular}{|c|c|c|c|c|c|c|}
\hline \multirow[b]{2}{*}{$w$ Pip infection } & \multirow[b]{2}{*}{$\mathrm{n}$} & \multirow[b]{2}{*}{ quinquefasciatus } & \multicolumn{4}{|c|}{ Culex pipiens taxa } \\
\hline & & & pipiens & molestus & pallens & $\overline{\text { Hybrids }}$ \\
\hline wPip-I & 171 & 133 & 16 & 20 & 0 & 2 \\
\hline wPip-II & 62 & 0 & 55 & 7 & 0 & 0 \\
\hline wPip-III & 96 & 46 & 30 & 20 & 0 & 0 \\
\hline wPip-IV & 31 & 0 & 15 & 15 & 1 & 0 \\
\hline wPip-V & 47 & 21 & 2 & 1 & 19 & 4 \\
\hline Undetermined & 2 & 0 & 1 & 1 & 0 & 0 \\
\hline
\end{tabular}




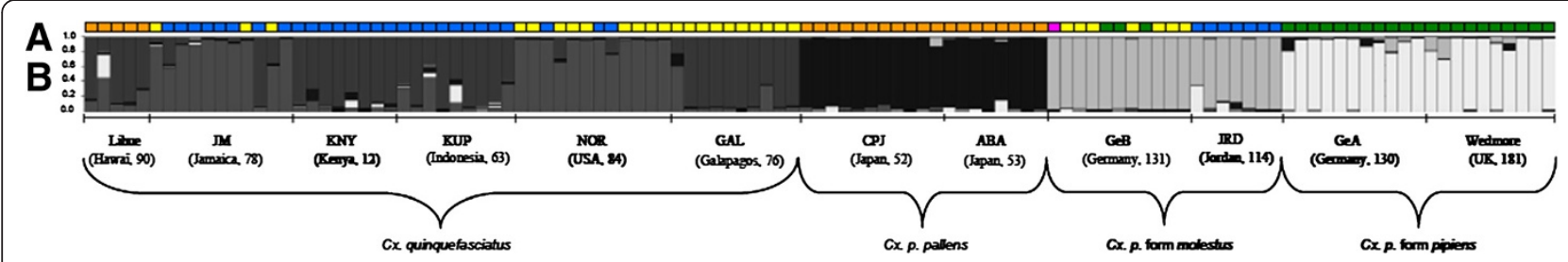

Figure 3 Comparison of wPip groups (A) with Cx. pipiens genetic clusters revealed by Bayesian analysis using microsatellites loci (B). Each of the 113 individuals included in the analysis is represented by a vertical line, partitioned into five squares assigned different colours (blue, wPip-I infection; green, wPip-II; yellow, wPip-III; pink, wPip-IV; orange, wPip-V) (A) and segments of different colours that represent the individual's probability of belonging to one of the four genetic clusters (black, pallens; medium and dark grey, quinquefasciatus; soft grey, molestus; white, pipiens) (B). Specimens were grouped by location (bracketed), and the indicated population numbers are the same as in Additional file 1: Table S1.

infection study. We further specifically tested for hostspecific associations by characterizing Wolbachia and mtDNA haplotypes using multilocus typing schemes.

The observed prevalence of $w$ Pip infection was 100\% in all $C x$. quinquefasciatus, $C x . p$. pipiens form pipiens, $C x . p$. pipiens form molestus and $C x . p$. pallens populations, as usually recorded for these $C x$. pipiens taxa $[8,25,42]$. The genotyping of $w$ Pip strains using the two ANK genes ank 2 and $p k 1$ confirmed the presence of five distinct $w$ Pip groups as observed in a previous study [14] showing that ank2 and $p k 1$ genes are suitable to assign $w$ Pip groups. However, by using additional $w$ Pip markers we detected greater diversity including new and recombinant haplotypes indicating higher $w$ Pip diversity than previously thought. Overall, our survey of infection diversity is therefore likely to seriously underestimate the true figure of $w$ Pip diversity that shows a rapid diversification in their natural host $C x$. pipiens.

The distribution of $w$ Pip groups appeared spatially structured, well exemplified by European $C x$. pipiens populations that harbour the highest $w$ Pip diversity across all examined geographic regions. This suggests that the $w$ Pip ancestor may have initially spread in
European populations where it evolved in five divergent groups, and that newly emerged $w$ Pip groups have only secondarily expanded outside Europe. Human migrations have also probably enhanced this process by expanding the geographic range of diverse $C x$. pipiens members. For instance, both $C x$. pipiens and $C x$. quinquefasciatus were recently introduced into the Americas and Australia $[16,17,19]$. In the Americas, the presence of two $w$ Pip groups indicates that at least two separate introductions have occurred: one probably from Europe introduced wPip-III-infected Cx. pipiens to North America, the other from tropical Africa or South Asia introduced $w$ Pip-Iinfected Cx. quinquefasciatus to Americas. In Australia, independent introduction events may also explain the presence of $w$ Pip-II and $w$ Pip- $\mathrm{V}$, otherwise mainly found in Western Europe and Asia. That American and Australian populations came from multiple and independent colonization events from Europe, Africa and Asia is also well supported by the examination of $C x$. pipiens microsatellites $[18,19]$. Overall, the $w$ Pip distribution is likely to result from ancient and recent imprints, underlining the importance of historical contingencies in the population structure of infections.

Table 4 Partitioning of cytb haplotypes between $C x$. pipiens taxa. Specimens with a hybrid signature were not included in these analyses

\begin{tabular}{|c|c|c|c|c|c|c|}
\hline \multirow[b]{2}{*}{ mtDNA_group } & \multirow[b]{2}{*}{ cytb haplotypes } & \multirow[b]{2}{*}{$\mathbf{n}$} & \multicolumn{4}{|c|}{ Culex pipiens taxa } \\
\hline & & & quinquefasciatus & pipiens & molestus & pallens \\
\hline \multirow[t]{4}{*}{ mtDNA_1 } & $\# 1$ & 1 & 1 & 0 & 0 & 0 \\
\hline & \#2 & 48 & 43 & 4 & 0 & 1 \\
\hline & \#3 & 18 & 10 & 3 & 5 & 0 \\
\hline & \#4 & 5 & 5 & 0 & 0 & 0 \\
\hline mtDNA_2 & \#5 & 38 & 0 & 35 & 3 & 0 \\
\hline \multirow[t]{2}{*}{ mtDNA_3 } & \#6 & 35 & 10 & 19 & 6 & 0 \\
\hline & \#7 & 2 & 1 & 1 & 0 & 0 \\
\hline \multirow[t]{2}{*}{ mtDNA_4 } & \#8 & 5 & 0 & 1 & 4 & 0 \\
\hline & $\# 9$ & 12 & 0 & 4 & 7 & 1 \\
\hline \multirow[t]{2}{*}{ mtDNA_5 } & $\# 10$ & 1 & 1 & 0 & 0 & 0 \\
\hline & $\# 11$ & 1 & 1 & 0 & 0 & 0 \\
\hline
\end{tabular}


Remarkably, the mtDNA variation in the $C x$. pipiens complex mirrored precisely the $w$ Pip variation, showing a great evidence for indirect selection arising from linkage disequilibrium with infections. mtDNA diversity was extremely reduced over the distribution area of $C x$. pipiens members, which is likely to be a consequence of cytoplasmic hitchhiking driven by the recent invasion of the $w$ Pip ancestor, as pointed by previous studies $[10,14,24]$. The mtDNA of $C x$. pipiens individuals infected by different $w$ Pip groups have further evolved to be distinct, showing that these two cytoplasmic elements have experienced a recent joint evolutionary history. As a result, $w$ Pip confounds the inference of $C x$. pipiens evolutionary history from mtDNA data, as often observed in other Wolbachiainfected species $[43,44]$.

Because of vertical transmission, one should expect that persistence of $w$ Pip infection in the Cx. pipiens complex over long periods of time should result in diversification of Wolbachia alongside the host (cocladogenesis). However, we did not observe this pattern: similar $w$ Pip groups and mtDNA haplotypes are found in different taxa and no cytoplasm type is specific to a given $C x$. pipiens taxa. We rather showed that the cytoplasmic diversity tends to be homogenized across the four members of the Cx. pipiens complex we examined. Collectively, the data suggest a recent, and possibly still ongoing, cytoplasmic exchange between Cx. pipiens taxa.

Two non-exclusive processes can explain why different $C x$. pipiens taxa share similar cytoplasms. The first process is the relatively recent emergence of some $C x$. pipiens taxa. The pipiens and molestus forms are very closely related and several scenarios place their divergence at 10,000 years ago [18], which is slightly more recent than the supposed emergence of $w$ Pip groups (estimated at ca 20,000 years ago; [14]). Assuming that $w$ Pip infections were initially present in the pipiensmolestus ancestor could explain why many shared $w$ Pip groups were found in both $C x$. pipiens taxa. The second process that could lead the homogenization of cytoplasmic diversity is linked to hybridization events within the complex. Although each member of the Cx. pipiens complex has an unique genetic signature, their genetic independence is not absolute since occasional inter-taxa hybridization may occur [16, 18, 22, this study]. Hybridization may transfer $w$ Pip infections and associated mtDNA from taxa to taxa (i.e. cytoplasmic introgression) [43]. This pattern fits well with the case of $C x$. quinquefasciatus. This taxon has emerged long before the pipiens-molestus divergence [18], and before the initial $w$ Pip infection in European populations. It is thus likely that $w$ Pip has secondarily spread from $C x$. pipiens to $C x$. quinquefasciatus following cytoplasmic introgression since recurring hybridization occurs where their distribution overlaps [16-18]. A similar process could also explain the presence of two different $w$ Pip groups in $C x . p$. pallens: this latter taxa is thought to be the result of unidirectional hybridization between $C x . p$. pipiens females and $C x$. quinquefasciatus males [45], a pattern that explains why $C x . p$. pipiens and $C x . p$. pallens share two $w$ Pip groups. Cytoplasmic introgression is likely still underway in the $C x$. pipiens complex: hybrids between pipiens and molestus forms have been documented in North America and in southern Europe $[18,22]$ as well as between $C x$. quinquefasciatus and Cx. p. pallens in Asia [45], and $w$ Pip infections may commonly flow from either side into the hybrid zone.

\section{Conclusions}

It is now clear that Wolbachia impacts the Cx. pipiens diversity in different ways. The $w$ Pip distribution presented here showed a well-structured picture, and underlines a critical example of cytoplasmic introgression through sibling taxa. This situation is actually similar to adaptive introgression of beneficial alleles. Previous studies examining loci involved in insecticide resistance concluded that resistant alleles are undergoing frequent expansion across the complex through hybridization between $C x$. pipiens members $[46,47]$. Survey of $C x$. pipiens neutral loci, however, show that recombination may break the connection between selected and neutral loci very quickly, maintaining the genetic differentiation between taxa and allowing the inter-taxa expansion of selected genes [19]. Hybridization can weakly impact the global flow of nuclear genes but serves as a powerful mechanism of rapid adaptation for insect populations through the penetration of useful adaptive alleles. The $w$ Pip infections seem to operate in a similar manner as insecticide resistance genes in the $C x$. pipiens members, suggesting that introgression has a crucial role in the dispersal of $w$ Pip infections. Of note, however, in the case of Wolbachia, infection spread may have a negative impact on population dynamics of mosquito hosts since it could drive deleterious mitotypes to fixation through cytoplasmic hitchhiking. It remains to be seen what role, if any, CI between incompatible $w$ Pip strains play in divergence between different geographic populations within the $C x$. pipiens complex.

\section{Additional file}

Additional file 1: Table S1. Detailed results of the screen of Culex pipiens populations. LL, laboratory lines; ND, not determined. Table S2. List of primers and gene features. Table S3. Nucleotide polymorphism in the cytb, ND2 and ND5 mitochondrial genes. Only polymorphic sites are represented and a dash indicates similarity with the top sequence. Position expressed in nucleotides bases on the complete mitochondrial sequence of Pel Culex pipiens line (Klasson et al. 2008). Table S4. 
Pair-wise FST values among mosquito populations (quinquefasciatus: populations 78, 12, 63, 84, 76 and 90 in Table S1, pallens: 52 and 53, molestus: 131 and 114, pipiens: 130 and 181). *, significant FST values after Bonferroni correction. Figure S1. Identification of ank2 and pk1 allelic profiles. (A) Hinfl digestion of the ank2 PCR products allowed discrimination of five alleles (a to e): a (one RFLP fragment: 313 bp), b $(217,195,98$ bp), c (293, 217 bp), d (217, 195 bp) and e (415 bp). (B) Taql digestion of the $p k 1$ PCR products allowed discrimination of four specific WPip alleles (alleles a and e have the same fragment sizes): a/e (903, 430 bp), b (669, 665 bp), c (851, 498 bp) and d (497, 251, 107 bp). (C) Pst/ digestion of the pk1 PCR products allowed discrimination alleles a (903, 303, 141 bp) and e (903, 430 bp). Figure S2. mtDNA phylogeny constructed using Maximum likelihood method based on concatenated sequences of cytb, ND2 and ND5 genes. mtDNA haplotypes originally described by Atyame et al.(2011b) are marked by full circles. Triangles show the seven specimens presenting incongruences between wPip infection and mtDNA haplotypes. Numbers on branches indicate percentage bootstrap support for major branches (500 replicates). The scale bar indicates the number of substitutions.

\section{Competing interests}

The authors declare that they have no competing interests.

\section{Authors' contributions}

Conceived and designed the experiments: ED, CMA, MW and OD. Performed the experiments: ED, CMA, OD, P Makoundou, SU, DMF, EVS. Analyzed the data: ED, P Milesi and OD. Wrote the paper: ED, MW and OD. All authors read and approved the final manuscript.

\section{Acknowledgments}

We are very grateful to N. Pasteur, F. Zélé, F. Tredez, J. Tonnabelle, S. Boukraa, C. Malcolm, M. Raymond, F. Rousset, N. Perrin, R. Dabire, G. Duvallet, P. Tortosa, P. Carnevale, P. Guillet, C. Ngoagouni, I. Dufour, J. Vontas, A. Tsagkarakou, D. Ayala, M Calviti, M. Toure, F. Cui, S. Marcombe and G. Müller for collection help. We are also grateful to N. Pasteur and P. Labbé, for their helpful comments and suggestions, and to A. Berthomieu for technical help. All sequence data were obtained on the Environmental Genomic Platform of the IFR Montpellier-Environnement-Biodiversité. Contribution 2013-x of the Institut des Sciences de l'Evolution de Montpellier (UMR 5554 CNRS Université Montpellier 2)

\section{Author details}

${ }^{1}$ Institut des Sciences de l'Evolution, UMR5554 CNRS, Université Montpellier 2 34095 Montpellier cedex 05, France. ²Université de la Réunion-CRVOI (Centre de Recherche et de Veille sur les Maladies Emergentes dans I'Océan Indien), 2 rue Maxime Rivière, 97490 Sainte Clotilde, France(lle de La Réunion)

${ }^{3}$ Center for Vector Biology, Rutgers University, 180 Jones Av, New Brunswick NJ 08901, USA. ${ }^{4}$ N.I. Vavilov Institute of General Genetics, Russian Academy of Sciences, Gubkin Str. 3, Moscow 119991, Russia.

Received: 23 May 2013 Accepted: 28 August 2013

Published: 3 September 2013

\section{References}

1. Duron O, Bouchon D, Boutin S, Bellamy L, Zhou L, Engelstadter J, Hurst GD: The diversity of reproductive parasites among arthropods: Wolbachia do not walk alone. BMC Biol 2008, 6:27.

2. Hilgenboecker $K$, Hammerstein $P$, Schlattmann $P$, Telschow A, Werren JH: How many species are infected with Wolbachia? a statistical analysis of current data. FEMS Microbiol Lett 2008, 281:215-220.

3. Zug R, Hammerstein P: Still a host of hosts for Wolbachia: analysis of recent data suggests that $40 \%$ of terrestrial arthropod species are infected. PloS one 2012, 7:e38544.

4. Werren JH, Baldo L, Clark ME: Wolbachia: master manipulators of invertebrate biology. Nat Rev Microbiol 2008, 6:741-751.

5. Engelstadter J, Hurst GD: What use are male hosts? the dynamics of maternally inherited bacteria showing sexual transmission or male killing. Am Nat 2009, 173:E159-E170.

6. Saridaki A, Bourtzis K: Wolbachia: more than just a bug in insects genitals. Curr Opin Microbiol 2010, 13:67-72.
7. Yen JH, Barr AR: New hypothesis of the cause of cytoplasmic incompatibility in Culex pipiens L. Nature 1971, 232:657-658.

8. Rasgon JL, Scott TW: Wolbachia and cytoplasmic incompatibility in the California Culex pipiens mosquito species complex: parameter estimates and infection dynamics in natural populations. Genetics 2003, 165:2029-2038.

9. Duron O, Bernard C, Unal S, Berthomieu A, Berticat C, Weill M: Tracking factors modulating cytoplasmic incompatibilities in the mosquito Culex pipiens. Mol Ecol 2006, 15:3061-3071.

10. Guillemaud T, Pasteur N, Rousset F: Contrasting levels of variability between cytoplasmic genomes and incompatibility types in the mosquito Culex pipiens. Proc Biol Sci 1997, 264:245-251.

11. Duron O, Boureux A, Echaubard P, Berthomieu A, Berticat C, Fort P, Weill M: Variability and expression of ankyrin domain genes in Wolbachia variants infecting the mosquito Culex pipiens. J Bacterio/ 2007, 189:4442-4448.

12. Duron O, Bernard J, Atyame CM, Dumas E, Weill M: Rapid evolution of Wolbachia incompatibility types. Proc Biol sci / R Soc 2012, 279:4473-4480.

13. Baldo L, Dunning Hotopp JC, Jolley KA, Bordenstein SR, Biber SA, Choudhury RR, Hayashi C, Maiden MC, Tettelin H, Werren JH: Multilocus sequence typing system for the endosymbiont Wolbachia pipientis. Appl Environ Microbiol 2006, 72:7098-7110.

14. Atyame CM, Delsuc F, Pasteur N, Weill M, Duron O: Diversification of Wolbachia endosymbiont in the Culex pipiens mosquito. Mol Biol Evol 2011, 28:2761-2772

15. Zélé F, Nicot A, Duron O, Rivero A: Infection with Wolbachia protects mosquitoes against Plasmodium-induced mortality in a natural system. J Evol Biol 2012, 25:1243-1252.

16. Vinogradova EB: Culex pipiens pipiens mosquitoes: taxonomy, distribution, ecology, physiology, genetics. Applied Importance and Control: Pensoft Publishers; 2000.

17. Farajollahi A, Fonseca DM, Kramer LD, Kilpatrick MA: "Bird biting" mosquitoes and human disease: a review of the role of Culex pipiens complex mosquitoes in epidemiology. Infect Genet Evol 2011, 2011:1577-1585.

18. Fonseca DM, Keyghobadi N, Malcolm CA, Mehmet C, Schaffner F, Mogi M, Fleischer RC, Wilkerson RC: Emerging vectors in the Culex pipiens complex. Sci 2004, 303:1535-1538.

19. Fonseca DM, Smith JL, Wilkerson RC, Fleischer RC: Pathways of expansion and multiple introductions illustrated by large genetic differentiation among worldwide populations of the southern house mosquito. Am J Trop Med Hyg 2006, 74:284-289.

20. Kent RJ, Harrington LC, Norris DE: Genetic differences between Culex pipiens f. Molestus and Culex pipiens pipiens (Diptera: Culicidae) in New York. J Med Entomol 2007, 44:50-59.

21. Miller BR, Crabtree MB, Savage HM: Phylogeny of fourteen Culex mosquito species, including the Culex pipiens complex, inferred from the internal transcribed spacers of ribosomal DNA. Insect Mol Biol 1996, 5:93-107.

22. Gomes B, Sousa CA, Novo MT, Freitas FB, Alves R, Côrte-Real AR, Salgueiro P, Donnelly MJ, Almeida APG, Pinto J: Asymmetric introgression between sympatric molestus and pipiens forms of Culex pipiens (Diptera: Culicidae) in the comporta region, portugal. BMC evol biol 2009, 9:262.

23. Irving-Bell RJ: Cytoplasmic factors in the gonads of Culex pipiens complex mosquitoes. Life Sci 1974, 14:1149-1151.

24. Rasgon JL, Cornel AJ, Scott TW: Evolutionary history of a mosquito endosymbiont revealed through mitochondrial hitchhiking. Proc Biol SCi 2006, 273:1603-1611.

25. Duron O, Lagnel J, Raymond M, Bourtzis K, Fort P, Weill M: Transposable element polymorphism of Wolbachia in the mosquito Culex pipiens: evidence of genetic diversity, superinfection and recombination. Mol Ecol 2005, 14:1561-1573.

26. Klasson L, Walker T, Sebaihia M, Sanders MJ, Quail MA, Lord A, Sanders S, Earl J, O'Neill SL, Thomson N, Sinkins SP, Parkhill J: Genome evolution of Wolbachia strain wPip from the Culex pipiens group. Mol Biol Evol 2008, 25:1877-1887.

27. Rogers SO, Bendich AJ: Extraction of DNA from plant tissues. In Plant molecular biology manuel. Volume A6. Edited by Gelvin SB, Schilperoort RA. Boston: Kluwer Academic Publishers; 1988:1-10.

28. Sambrook J, Fritsch EF MT: Molecular cloning: a laboratory manual. Cold Spring Harbor, NY: Cold Spring Harbor Laboratory Press; 1989.

29. Bourguet D, Foncesca D, Vourch G, Dubois MP, Chandre F, Severini C Raymond M: The acetylcholinesterase gene ace: a diagnostic marker of the pipiens and quinquefasciatus forms of the Culex pipiens complex. J Amer Mosq Control Assoc 1998, 14:390-396. 
30. Smith JL, Fonseca DM: Rapid assays for identification of members of the Culex (Culex) pipiens complex, their hybrids, and other sibling species (Diptera: culicidae). Am J Trop Med Hyg 2004, 70:339-345.

31. Bahnck CM, Fonseca DM: Rapid assay to identify the two genetic forms of Culex (Culex) pipiens L. (Diptera: Culicidae) and hybrid populations. Am J Trop Med Hyg 2006, 75:251-255.

32. Thompson JD, Gibson TJ, Higgins DG: Multiple sequence alignment using ClustalW and ClustalX. Curr Protoc Bioinform 2002, Chapter 2:Unit 23. http://onlinelibrary.wiley.com/doi/10.1002/0471250953.bi0203s00/full.

33. Kumar S, Tamura K, Nei M: MEGA3: integrated software for molecular evolutionary genetics analysis and sequence alignment. Brief Bioinform 2004, 5:150-163.

34. Castresana J: Selection of conserved blocks from multiple alignments for their use in phylogenetic analysis. Mol Biol Evol 2000, 17:540-552.

35. Tajima F, Nei M: Estimation of evolutionary distance between nucleotide sequences. Mol Biol Evol 1984:269-285.

36. Nei M, Kimura S: Molecular evolution and phylogenetics. Oxford New York: Oxford University Press; 2000.

37. Raymond M, Rousset F: Genepop (version 1.2), A population genetics software for exact tests and ecumenicism. J Hered 1995, 86:248-249.

38. Hubisz MJ, Falush D, Stephens M, Pritchard JK: Inferring weak population structure with the assistance of sample group information. Mol ecol res 2009, 9:1322-1332.

39. Evanno G, Regnaut $\mathrm{S}$, Goudet J: Detecting the number of clusters of individuals using the software STRUCTURE: a simulation study. Mol ecol 2005, 14:2611-2620

40. Keyghobadi N, Matrone MA, Ebel GD, Kramer LD, Fonseca DM: Microsatellite loci from the northern house mosquito (Culex pipiens), a principal vector of West Nile virus in North America. Mol Ecol Notes 2003, 4:20-22.

41. Fonseca DM, Atkinson CT, Fleischer RC: Microsatellite primers for Culex pipiens quinquefasciatus, the vector of avian malaria in Hawaï. Mol Ecol 1998, 7:1617-1619.

42. Duron O, Raymond M, Weill M: Many compatible Wolbachia strains coexist within natural populations of Culex pipiens mosquito. Heredity 2011, 106:986-993.

43. Hurst GDD, Jiggins FM: Problems with mitochondrial DNA as a marker in population, phylogeographic and phylogenetic studies: the effects of inherited symbionts. Proc Biol Sci 2005, 272:1525-1534.

44. Charlat S, Duplouy A, Hornett EA, Dyson EA, Davies N, Roderick GK, Wedell N, Hurst GD: The joint evolutionary histories of Wolbachia and mitochondria in Hypolimnas bolina. BMC Evol Biol 2009, 9:64.

45. Fonseca D, Smith J, Kim H, Moggi M: Population genetics of the mosquito Culex pipiens pallens reveals sex-linked asymmetric introgression by Culex quinquefasciatus. Infect Genet Evol 2009, 2009:1197-1203.

46. Raymond M, Callaghan A, Fort P, Pasteur N: Worldwide migration of amplified insecticide resistance genes in mosquitoes. Nature 1991, 350:151-153.

47. Labbe P, Lenormand T, Raymond M: On the worldwide spread of an insecticide resistance gene: a role for local selection. J Evol Biol 2005, 18:1471-1484

doi:10.1186/1471-2148-13-181

Cite this article as: Dumas et al:: Population structure of Wolbachia and cytoplasmic introgression in a complex of mosquito species. BMC Evolutionary Biology 2013 13:181.

\section{Submit your next manuscript to BioMed Central and take full advantage of:}

- Convenient online submission

- Thorough peer review

- No space constraints or color figure charges

- Immediate publication on acceptance

- Inclusion in PubMed, CAS, Scopus and Google Scholar

- Research which is freely available for redistribution

Submit your manuscript at www.biomedcentral.com/submit
C Biomed Central 